\title{
Drug Delivery and Hydrotropism
}

\author{
Dr. Sunil Kulkarni* \\ Chemical Department, Gharda Institute of Technology, Lavel.Tal.Khed.Dist.Ratnagiri, Mumbai University
}

*Corresponding Author: Dr. Sunil Kulkarni, Chemical Department, Gharda Institute of Technology, Lavel.Tal.Khed.Dist.Ratnagiri, Mumbai University

\begin{abstract}
Drug delivery systems play major role in the utilization of drugs. The mode of injection of the drug plays a major role is deciding ease of drug delivery. For the pharmaceutical compounds, it is very important to have proper drug delivery system which will keep their effect intact during the transportation in side our body. The concentration of the drug inside the body needs to be maintained above certain value to get effect out of the drug. For this purpose, the drug intake is given three to four times a day to the patient. The ability of the person to follow the drug intake schedule and the severity of the drug requirement decides mode of drug delivery which in turn has major role to play in drug transportation. The solubility of the compound in the solvent plays important role in utilization of the compound for drug delivery. Many common drugs are injected into body by oral route through gastrointestinal tracts. Many of the drugs are susceptible to the enzymatic degradation and hence cannot be injected orally. Such drugs are administered through needles. Increasing solubility of the compound can be done by using surfactants, pH adjustments, size reduction, salt formation. Each method has some merits and demerits. Hydrotropism is one of the very useful method for increasing solubility of the drugs.
\end{abstract}

Keywords: Solubilization, bioavailability, injection, carriers, concentration

\section{INTRODUCTION}

\subsection{Biotechnology and Drug Delivery System}

Biotechnology is one of the important domains in chemical engineering. As they say, the best processing plant possible is our body system. Almost all the mechanical operations, Reactions (biochemical), separations, downstream processing occur in our body system. Kinetics of biological reactions, enzyme kinetics, gene therapy, recombinant technology, drug delivery are some of the topics in biotechnology. Also, for chemical engineering courses, biotechnology is one of the electives in final year students in India in many universities. It is natural for a chemical engineer to take interest in biotechnology. Drug delivery systems play major role in the utilization of drugs. The mode of injection of the drug plays a major role is deciding ease of drug delivery. For the pharmaceutical compounds, it is very important to have proper drug delivery system which will keep their effect intact during the transportation in side our body [1-4].

\section{THE MECHANISM}

The concentration of the drug inside the body needs to be maintained above certain value to get effect out of the drug. For this purpose, the drug intake is given three to four times a day to the patient. The ability of the person to follow the drug intake schedule and the severity of the drug requirement decides mode of drug delivery which in turn has major role to play in drug transportation. Whenever drug is injected in the bodies, it spreads across various parts of bodies. Its presence is normally undesired in all parts of body. Many investigations are reported on the localized and specific drug delivery systems. In any case, this aspect reduces the amount of drug required also. Localized and specific drug delivery has advantages such as fast and rapid effects and immediate and fast relief for the patient. Diffusion plays very important role in drug delivery. Many time affinities-based mechanisms are used. The concept of hydrotropism plays very significant role in drug delivery [5-8].

\subsection{Solubility of a Drug}

The solubility of the compound in the solvent plays important role in utilization of the compound for drug delivery. Many common drugs are injected into body by oral route through gastrointestinal 
tracts. Many of the drugs are susceptible to the enzymatic degradation and hence cannot be injected orally. Such drugs are administered through needles. Increasing solubility of the compound can be done by using surfactants, $\mathrm{pH}$ adjustments, size reduction, salt formation. Each method has some merits and demerits. Hydrotropism is one of the very useful method for increasing solubility of the drugs [9-12].

\subsection{Hydrotropism}

There are some compounds which helps hydrophobic compounds to solubilize by the mechanism of micellar solubilization. This phenomenon is known as hydrotropism. Enhancement in solubility of various compounds in water for drug formulation is most significant application of hydrotropy. Also, the 'hydrotropy' concept is very useful for analysis in the pharmaceutical industries. For rapid and accurate results, the concept of hydrotropy can be coupled with spectrophotometry and chromatography. This article provides insight into the application of hydrotropy in various applications in medical and pharmaceutical applications [13-16].

\subsection{Hydrotropism and Drug Delivery System}

Tyagi et al. carried out a review on hydrotropism for enhancement of solubilization and bioavailability of poorly soluble drugs [17]. They discussed solubility various enhancement methods.

According to them, there are various methods for alteration of the solubility and hydrotropy can be used very effectively for the purpose. Factors like drug property, site of absorption, and required dosage form and characteristics influence selection of method to be used for solubility enhancement.

The importance of hydrotropy in solubility enhancement was explained by Ali and Choudhary [18]. They pointed out that that about 40 percent drugs are water insoluble and hence it is very important to use easy and applicable method for increasing the solubility of drugs. They highlighted the importance of hydrotropy in the pharmaceutical and threptic applications

Dhinakaran, et.al. Carried out separation of $\mathrm{m} / \mathrm{p}$-aminoacetophenone with the application of hydrotropy principle [19]. They investigated the separation by using diethyl nicotinamide,sodium pseudo cumene sulfonate and sodium thiocyanate solutions as hydrotropes. According to this investigation, percentage separation of maminoacetophenone from $\mathrm{m} / \mathrm{p}$-aminoacetophenone increased with concentration of hydrotropes.

Combination of conventional analysis methods like spectrophotometer and chromatography can be used for analysis with hydrotropes. Most of these methods were proven to be accurate, rapid and precise. The solubility enhancement was observed in some cases. In most of the investigations, it was observed that the solubility increased with concentrations [20].100-200-fold increase in the solubility was observed in some cases.

\section{RESEARCH ON DRUG DELIVERY SYSTEMS WITH EMPHASIS ON GASTRIC DRUGS}

The drug delivery is important area of investigation in modern medical and pharmaceutical advancements. Oral drug delivery is most widely practiced drug delivery system. In the current methods for drug delivery, the drug has to be administered several times in order to retain its therapeutic effects. The results of this methods show large fluctuations in the drug level. Hafeez et al. carried out investigation on new drug delivery system in order to overcome this problem [21]. In their method, it needed only single dose which released active ingredients over the period of time. Also, the active entity is directly delivered on the specific site and thus the side effects are avoided. The release of the active agent maintains the drug level almost constant over the period of time. Thus, therapeutic concentration is maintained for sustained period of time. Various drug release methods and drug forms are being investigated due to their medicinal importance. In case gastric drugs, the most important aspect is control on placement of a variety of important drugs through appropriately designed drug delivery system (DDS) in a specific region[21].Prolonged retention of drug in gastrointestinal tract bioavailability, reduces drug waste and improves solubility of drugs that are less soluble in a high $\mathrm{pH}$ environment[21].This type of gastroretentative drug delivery system can developed based on various principals. Floating drug delivery system is based on buoyancy principle. Bio adhesive systems, Swelling and expanding systems and High-density systems with their physicochemical advantages can be used for gastric retention. Floating microspheres is very efficient tool for drug delivery. 
Homayun et al. carried out investigation on recent developments on drug delivery systems [22]. According to them, route of drug delivery systems and their characteristic play very significant role in drug delivery systems. Long shelf life, sustained delivery, ease of administration and intensified immune response are attributes of oral drug delivery systems, which make them the most popular choice for drug delivery. There are some obstacles in oral drug delivery systems, which needed to be removed. One of the disadvantages of oral route is that the absorption mechanism is very complicated.Oral carriers deal with number of biological barriers. It is also necessary to avoid other destructive effects such as contact with organic solvents; shear stresses and local temperature increases on molecules.

Pharmaceutical, medical and biological industries are largely dependent on gases used in the analytical methods and liquids used as solvent or carriers. It is very critical to removal impurities from these fluids and also avoid them from entering these fluids. According to Akerlindh and Ahamparam, maintaining the purity of these fluids from manufacturing facilities to the point of application is also a challenging task [23]. Good interpretation of available data, preparing and following the protocols and executionof standard operating procedures can help in maintain the integrity of these gases.

Various in-vitro systems can be used to accurately control drug delivery system. Gastric retention time is controlled by factors such as Size of the dosage form, Density of Dosage Form, Shape of the dosage form Viscosity grade of polymer. In addition to this, female has slower gastric emptying rate (4.6 \pm 1.2 hours) than males (3.4 \pm 0.6 hours) [24]. Prasanth et al. studied colon specified drug delivery system [25]. Colon is site where drug delivery takes place. In this drug is directly targeted towards the infected colon. Due to this the drug lose is lowered. The drugs with limited solubility, instability and selective applications are the candidates for research as these are unsuitable for gastroretentive drug delivery systems [26]. To achieve gastric retention, floating and non-floating drug delivery systems can be used. THE density of these drugs is higher than normal stomach content in order to increase residence time. The bulk density of such drugs in lo than the other contents so that it is buyout and floats on the surface. According to Yadav and Verma, pharmaceutical pellets are versatile carrier for oral controlled delivery of drugs [27]. Different palatizing techniques like layering, extrusion spherization, cryopelletization, hot melt extrusion can be used for pellet formation. The agglomeration formation and growth of pellet can be explained in terms of mechanism that includes nucleation, coalescence and layering, ball growth phase. The coating on the pellets helps to mask the taste, odour, and colour of the drug. It also It avoids inactivation of drugs in stomach.

\section{CONClusion}

Drug delivery systems play major role in the utilization of drugs. Metabolism of the drug in human and animal bodies is still being studied [28]. Simpler and effective drug delivery systems can be explored with thorough investigation on metabolism [29]. Oral drug delivery faces challenges in terms of effectiveness, cost, side effects and formulation [30]. The drugs with limited solubility, instability and selective applications are the candidates for research as these are unsuitable for gastroretentive drug delivery systems. To achieve gastric retention, floating and non-floating drug delivery systems can be used. The density of these drugs is higher than normal stomach content in order to increase residence time. The mode of injection of the drug plays a major role is deciding ease of drug delivery. For the pharmaceutical compounds, it is very important to have proper drug delivery system which will keep their effect intact during the transportation in side our body. There are some compounds which helps hydrophobic compounds to solubilize by the mechanism of micellar solubilization. Combination of conventional analysis methods like spectrophotometer and chromatography can be used for analysis with hydrotropes. Most of these methods were proven to be accurate, rapid and precise. The solubility enhancement of was observed in some cases. In most of the investigations, it was observed that the solubility increased with concentrations [20].100-200-fold increase in the solubility was observed in some cases

\section{REFERENCES}

[1] G.Chawla, P. Gupta, V. Koradia, A,K. Bansal, "Gastro retention: A Means to Address Regional Variability in Intestinal Drug Absorption", Pharmaceutical Technology, pp. 50-52, 2003.

[2] S. H. Shaha, J.K. Patel, K. Pundarikakshudu, "An overview of a gastro-retentive floating drug delivery system”, Asian Journal of Pharmaceutical Sciences, Vol.4, No.1, pp.65-80, 2009.

[3] B.Y.Choi, H.J.Park, S.J.,Hwang, J.B.,, "Preparation of alginate beads for floating drug delivery: effects of $\mathrm{CO}_{2}$ gas forming agents", Int. J. Pharm., Vol.239, 81-91, 2002. 
[4] S.H.Shah, J.K.Patel, N.V. Patel, "Stomach specific floating drug delivery system: a review”, Int J Pharm Res, Vol. 1, No.3, 623-633, 2009.

[5] S.T.Kumar, N.N.Gandhi, "Association model of hydrotropy for the effect of hydrotropes on solubility and mass transfer coefficient of acetylsalicylic acid”, Int J Pharm Pharm Sci., Vol.3, No.4, 600-605, 2012.

[6] Kapadiya Nidhi, SinghviIndrajeet, Mehta Khushboo, Karwani Gauri, and Dhrubo Jyoti Sen, "Hydrotropy: A Promising Tool For Solubility Enhancement:A Review", International Journal Of Drug Development \& Research, Vol. 3, No. 2, pp26-34,2011.

[7] J.K. Badjatya, R. B. Bodla, U. B. Moon, "Enhancement Of Solubility Of Fenofibrate By Using Different Solubilization Techniques”, Asian Journal Of Pharmacy \& Life Science, Vol. 1, No.2, pp.144-148, 2011.

[8] Shailendra Pandey, R.K. Maheshwari, “A Novel Spectroscopic Method For Estimation Of Ketoprofen In Tablet Dosage Form Using Hydrotropic Solubilization Methods", World Applied Sciences Journal, Vol.11, No.12, pp. 1524-1527, 2010.

[9] Sunil Jayant Kulkarni, AjaygiriKamalgiri Goswami, "Research on Application of Hydrotropy: A Review", International Journal of Science, Engineering and Technology Research, Vol.3, No. 10, 2278 - 7798 , 2014.

[10] V. Pareek, S. R. Tambe And S. B. Bhalerao, "Role Of Different Hydrotropic Agents In Spectrophotometric And Chromatographic Estimation Of Cefixime", International Journal Of Pharma And Bio Sciences, Vol.1, No.3, pp.1-10, 2010.

[11] Natarajan Arunodhaya, Chinnakannu Jayakumar And Nagarajan Nagendra Gandhi, "Effect Of Hydrotropes On Solubility And Mass Transfer Coefficient Of Chlorobenzene", Research Journal Of Chemical Sciences, Vol. 2, No.8, pp.9-13, 2012.

[12] P. A. Winsor, "Hydrotropy, Solubilisation And Related Emulsification Processes", Trans. Faraday Soc., Vol.44, pp.376-398,1948.

[13] JayroniaSonali, Yadav Kamaldeep, Sharma Bhumika, Jain Sanjay, Maheshwari Rajesh Kumar, "Hydrotropy: A Novel Approach In Estimation Of Poorly Aqueous Soluble Drugs By TLC", International Journal of Pharmacy and Pharmaceutical Sciences, Vol. 5, No. 2, pp.176-178, 2013.

[14] S.T.Kumar, N.N. Gandhi, "Association model of hydrotropy for the effect of hydrotropes on solubility and mass transfer coefficient of acetylsalicylic acid”, Int J Pharm Pharm Sci., Vol.3, No.2, pp.600-605, 2012.

[15] A.M.Saleh, L.K. El-Khordagui, "Hydrotropic agent a new definition", International journal of pharmaceutics, Vol.24, pp.231-238, 1985.

[16] A.Darwish, A.T.Florence and A.M. Saleh, "Effects of hydrotropic agents on the solubility, precipitation, and protein binding of etoposide", J. Pharm. Sci., Vol.78, 1989.

[17] Prof. Satyanand Tyagi, Patel Chirag J, DadarwalPoonam,Mangukia Dhruv, Sojitra Ishita, Bhupender Kumar Nimbiwal, Virendra Singh,Dr. K.V. Subrahmanyam, "A Novel Concept For Enhancement of Solubilization And Bioavailability of Poorly Soluble Drugs: Hydrotropy: A Review”, Int. Journal Of Pharmaseutical And Bioscience, Vol. 2, No.1, pp.372-381, 2013.

[18] Md. Sajid Ali, Vicky Choudhary, "Solubility Enhancement Methods With Importance of Hydrotropy", Journal of Drug Delivery and Therapeutics, Vol 2, No. 6, 2012.

[19] M. Dhinakaran, Antony Bertie Morais and N. Nagendra Gandhi, "Separation Of M/P-Aminoacetophenone Using Hydrotropy”, E-Journal Of Chemistry”, Egyptian Journal of Chemistry, Vol.9, No.4, pp.2006-2014, 2012.

[20] Ji Young Kim Sungwon Kim, Michelle Papp, Kinam Park, Rodolfo Pinal, "Hydrotropic Solubilization of Poorly Water-Soluble Drugs”, Journal of Pharmaceutical Sciences, Vol. 99, No. 9, pp.3953-3965, 2010.

[21] Abdul Hafeez, Arun Maurya, Jagpal Singh, Ankit Mittal, Lakhan Rana, "An overview on floating microsphere: Gastro Retention Floating drug delivery system (FDDS)", The Journal of Phytopharmacology, Vol.2, No.3, pp.1-12, 2013.

[22] Bahman Homayun, Xueting Lin and Hyo-Jick Choi, "Challenges and Recent Progress in Oral Drug Delivery Systems for Biopharmaceuticals”, Pharmaceutics, Vol.11, No.3, 2019; doi: doi: 10.3390/pharmaceutics11030129.

[23] Katrin Åkerlindh and ShivanAhamparam, "Maximising Drug Discovery by Simplifying Scale-up and Production”, Drug: Discovery, Discovery- Specialty Gases, Touch Briefings, pp.57-61, 2009.

[24] Shashi Kiran Misra, 'Gastrointestinal targeting drug delivery system: A Review' , Journal of Pharmacy Research, Vol.4, No.8, pp.2751-2754, 2011.

[25] V.V.Prasanth, R.Jayaprakash, Sam T. Mathew, "Colon Specific Drug Delivery Systems: A Review on Various Pharmaceutical Approaches", Journal of Applied Pharmaceutical Science, Vol.2, No.1, pp.163169, 2012. 
[26] Amit Kumar Nayak, Ruma Maji, Biswarup Das, "Gastroretentive drug delivery systems: a review", Asian Journal of Pharmaceutical and Clinical Research, Vol.3, No. 1, pp.2-10, 2010.

[27] Niti Yadav and Anurag Verma, "Pharmaceutical Pellets: A Versatile Carrier for Oral Controlled Delivery of Drugs", Indian Journal of Pharmaceutical Education and Research, Vol. 50, No. 3, pp.146-160, 2016.

[28] Corina Ionescu, Mino R. Caira, "Drug Metabolism Current Concepts", Published by Springer, pp.1-37, 2005.

[29] HodaVarasteghan, Javad Shokri, SolmazAsnaashari and Yousef Javadzadeh, "Formulation and Evaluation of Novel Bilayer Floating and Sustained Release Drug Delivery System of Diltiazem HCl”, Int J Drug Dev and Res., Vol.10, No.4, pp. 1-3, 2019.

[30] Katherine Brake, Ashwini Gumireddy, Amit Tiwari, Harsh Chauhan and Dunesh Kumari, "In vivo Studies for Drug Development via Oral Delivery: Challenges, Animal Models and Techniques", Pharm Anal Acta, Vol.8, No.9, pp.1-11, 2017. DOI: 10.4172/2153-2435.1000560

Citation: Dr. Sunil Kulkarni, "Drug Delivery and Hydrotropism”, International Journal of Advanced Research in Chemical Science, 7(2), pp. 1-5. DOI: http://dx.doi.org/10.20431/2349-0403.0702001

Copyright: (C) 2020 Authors, This is an open-access article distributed under the terms of the Creative Commons Attribution License, which permits unrestricted use, distribution, and reproduction in any medium, provided the original author and source are credited. 